\title{
Evolution of a transposon in Daphnia hybrid genomes
}

Roland Vergilino ${ }^{1,2,3}$, Tyler A Elliott ${ }^{4}$, Philippe Desjardins-Proulx ${ }^{1,5}$, Teresa J Crease ${ }^{4^{*}}$ and France Dufresne ${ }^{1,2}$

\begin{abstract}
Background: Transposable elements play a major role in genome evolution. Their capacity to move and/or multiply in the genome of their host may have profound impacts on phenotypes, and may have dramatic consequences on genome structure. Hybrid and polyploid clones have arisen multiple times in the Daphnia pulex complex and are thought to reproduce by obligate parthenogenesis. Our study examines the evolution of a DNA transposable element named Pokey in the D. pulex complex.

Results: Portions of Pokey elements inserted in the $28 \mathrm{~S}$ rRNA genes from various Daphnia hybrids (diploids and polyploids) were sequenced and compared to sequences from a previous study to understand the evolutionary history of the elements. Pokey sequences show a complex phylogenetic pattern. We found evidence of recombination events in numerous Pokey alleles from diploid and polyploid hybrids and also from non-hybrid diploids. The recombination rate in Pokey elements is comparable to recombination rates previously estimated for $28 \mathrm{~S}$ rRNA genes in the congener, Daphnia obtusa. Some recombinant Pokey alleles were encountered in Daphnia isolates from multiple locations and habitats.
\end{abstract}

Conclusions: Phylogenetic and recombination analyses showed that recombination is a major force that shapes Pokey evolution. Based on Pokey phylogenies, reticulation has played and still plays an important role in shaping the diversity of the D. pulex complex. Horizontal transfer of Pokey seems to be rare and hybrids often possess Pokey elements derived from recombination among alleles encountered in the putative parental species. The insertion of Pokey in hotspots of recombination may have important impacts on the diversity and fitness of this transposable element.

Keywords: Daphnia pulex, Transposable element, Pokey, Hybrids, Recombination

\section{Background}

Transposable elements (TEs) are widespread in the living world. Few multicellular eukaryotes lack these genetic elements, which have the ability to move and multiply throughout the genome [1], but see [2] for exceptions in endosymbiont genomes. There is substantial variation in the proportion of mobile genetic elements and especially TEs across genomes [3]. Numerous studies have explored whether TE history reflects the evolutionary history of their hosts (that is, co-evolution [4-7]). Due to their high densities in eukaryotic genomes and their irreversible mode of insertion, some authors have proposed that SINEs (Short INterspersed Elements, non-autonomous Class I TEs) may be used for inferring the evolutionary

\footnotetext{
*Correspondence: tcrease@uoguelph.ca

${ }^{4}$ Department of Integrative Biology, University of Guelph, Science complex, Guelph, ON N1G 2 W1, Canada

Full list of author information is available at the end of the article
}

history of their host [8]. The phylogeny of a strictly vertically transmitted TE (from parents to offspring) should reflect that of its host(s). However, genetic and evolutionary processes, which may occur during speciation, may affect subsequent phylogenetic inferences based on TE sequences. For example, horizontal transfer (that is, transmission by vectors unrelated to host reproduction), incomplete lineage sorting (that is, the maintenance of ancient polymorphism in closely related species), lack of genetic variability or hybridization/introgression may account for incongruent phylogenetic patterns between TEs and their hosts [9-13]. Hybrid genomes should carry TE(s) from the genomes of their parental species. In contrast, if horizontal transfers occur between species that share ecological niches or are infected by the same parasites, we do not expect that TE phylogenies will match species phylogenies.

\section{Biomed Central}


The Daphnia pulex complex has been intensively studied due to its dominance in freshwater habitats in North America, and its variation in reproductive mode and ploidy level. Daphnia usually reproduce by cyclic parthenogenesis, which is clonal reproduction interrupted by bouts of sexual reproduction. However, some lineages reproduce by obligate parthenogenesis [14-18]. The D. pulex complex includes seven species that have been distinguished on the basis of morphological, ecological, and genetic data [19-22]. In North America, two species of the complex, D. pulex and Daphnia pulicaria, are dominant in freshwater habitats, and are morphologically similar but ecologically distinct [19], although they hybridize in nature [18,23-25]. Diploid hybrids always have D. pulex mitochondrial genomes (mtDNA) and have been found to reproduce by obligate parthenogenesis in nature based on patterns of allozyme variation $[18,26]$ and laboratory crosses [26], but see [27] for experimentally produced hybrids capable of sexual reproduction. Analyses of allozyme variation indicate that introgression is rare or nonexistent in areas where the two species co-occur $[16,18,25]$ but a recent study using mitochondrial and nuclear markers has shown that introgression between these species had a substantial impact on their evolutionary history [28].

Hybridization has also occurred among other species in the D. pulex complex [29-32]. For example, hybridization between North American species and circumarctic or European Daphnia species has been suggested based on allozyme, mtDNA or nuclear markers such as the ribosomal intergenic spacer (IGS) [21,33,34]. Some hybrids found in arctic and subarctic regions are polyploids (mainly triploids [35]). Polyploids with D. pulex, D. pulicaria and Daphnia middendorffiana (a circumarctic species) mtDNA are thought to have arisen from hybridization between $D$. pulex and $D$. pulicaria, or with another species which no longer exists as a cyclic parthenogen [22,29,30,32]. Another group of polyploids has been found in Daphnia tenebrosa, a circumarctic species [31] and includes both triploid and tetraploid clones [35] but their hybrid nature is unknown [22].

The D. pulex genome has recently been sequenced [36] and numerous Class II DNA transposons have been identified from it [37]. One Class II element, Pokey (Subclass I; sensu [38]) of the piggyBac superfamily, has been extensively studied in non-hybrid populations. It inserts in a site-specific fashion into a unique TTAA site in the tandemly repeated $28 \mathrm{~S}$ rRNA genes [39-41] and is also encountered in other parts of the genome. Two Pokey elements from $D$. pulicaria have been completely sequenced [39] and show that the element has 16 bp imperfect terminal inverted repeats (TIRs) and encodes a putative transposase that is much longer than originally estimated and contains an intron (Y. Bigot, personal communication, [40]). In addition, the non-coding region upstream of the transposase gene contains a repeated sequence that is similar to a sequence in the unique region of the host's ribosomal IGS [34,39,40]. Based on patterns of polymorphism observed among natural populations, previous studies $[41,42]$ have suggested that Pokey may be active in sexual populations of $D$. pulex but not in obligate parthenogens. Although Class II DNA transposons are more prone to transfer horizontally between lineages than retrotransposons, horizontal transfer does not seem to play a major role in the evolution of Pokey as 28S rRNA genes and mtDNA phylogenies of species in the subgenus Daphnia are congruent with the phylogeny of Pokey elements [7] leading to the conclusion that Pokey elements may co-evolve with their hosts.

The purpose of the present study is to describe Pokey sequences in hybrid isolates from the $D$. pulex complex. Specifically, we aim to determine if Pokey sequences inserted in rRNA genes reflect the putative parental species origins of the hybrid isolates. As Pokey elements insert in $28 \mathrm{~S}$ rRNA genes, they are subject to genetic processes such as inter-allelic recombination, gene conversion and unequal crossing over [43] that may lead to the concerted evolution of these genes. If Pokey elements are strictly vertically inherited and if recombination is negligible, we expect that a phylogeny of Pokey elements should follow the host phylogeny, and Pokey elements encountered in hybrid genomes should correspond to those encountered in their host parental species. If recombination is important in Pokey evolution and has occurred between elements showing recent activity and after hybridization, hybrid isolates will carry either sequences inherited from both parents or mosaic sequences between parental types. Alternatively, if recombination occurs between inactivated elements that diverged in the distant past, we could see Pokey mosaic sequences that do not necessarily reflect the progenitor species of the hybrid isolates in the D. pulex complex.

After cloning and sequencing partial Pokey elements from representatives of species in the $D$. pulex complex, we: (a) detected recombinant elements and located the recombination breakpoints in each, (b) estimated recombination rate parameters across sequences using a coalescent based method, (c) determined parental sequences from which the recombinant elements arose and (d) reconstructed the evolutionary history of the Pokey sequences. Moreover we used a PCR-RFLP protocol to search for the presence of different Pokey elements in additional hybrid and non-hybrid isolates of the D. pulex complex across freshwater habitats and geographical locations.

\section{Methods}

Daphnia samples

We analyzed 51 isolates representing six of the seven lineages of the $D$. pulex complex identified by Colbourne 
et al. [20]. Only D. melanica was not included. Nine Pokey sequences were obtained from nine isolates studied by Penton and Crease [7]. We established parthenogenetic lines (isolates) in the laboratory from 42 individual females sampled from nature between 2004 and 2010 (see Additional file 1) and cultured them using standard techniques [44]. For 50 of these isolates, genomic DNA was extracted from 10 to 30 individuals weighing approximately $100 \mathrm{mg}$ (wet weight) using the DNeasy Tissue kit (QIAGEN Inc., Mississauga, ON, Canada) according to the supplier's protocol. Species identity of these isolates was assessed by combining information on morphology, haplotype of the mitochondrial ND5 gene and genotype of the nuclear Lactate dehydrogenase ( $L d h)$ gene as described in [22]. Ploidy levels were previously assessed using microsatellite genotypes and flow cytometry [35].

\section{Structure of the Pokey transposase gene}

RT-PCR was performed on RNA samples extracted from a sexual $D$. pulex isolate to determine if Pokey transposase transcripts were present and to provide further evidence for the presence of an intron in the transposase gene. RNA was extracted from the parthenogenetic offspring of one sexual D. pulex isolate PX2-ON-9 (provided by Dr. M. Cristescu at the University of Windsor). The tissue was stored in RNA Later (Qiagen) at $-20^{\circ} \mathrm{C}$ and RNA was extracted using the RNAqueous4PCR kit (Life Technologies Inc., Grand Island, NY, USA) and standard manufacturer's protocols. The absence of DNA contamination was verified using standard PCR and Pokey transposase primers. Reverse transcription of the RNA used the SuperScript III One-Step RTPCR with Platinum Taq Kit (Life Technologies) and standard manufacturer's protocols. Primers used were Pok4065F (5'-TGATTCACCGAGGCCTCAGTTC) with Pok4488R (5'-GAATCGCTCGCGAGTCATGG) and Pok5026F (5'-TCGAACCTGCAGCCGGACGAATTTG CAG) with Pok5985R (5'-CACGTCGGTTAGAATATTC TGGCTCGTCGG). Numbers correspond to nucleotides in the $6.6 \mathrm{~kb}$ element from D. pulicaria (GenBank accession \# AY115589.1, [8]).

\section{Cloning and sequencing Pokey alleles}

To examine the impact of hybridization on Pokey diversity, Pokey alleles inserted in 28S rRNA genes from various isolates of the $D$. pulex complex were cloned and sequenced. An approximately 1600 bp fragment of Pokey from 16 isolates (2 diploid hybrids, 5 polyploid hybrids, 1 introgressed polyploid (TE3-MB-4), 3 diploids, 3 polyploids with unknown hybrid state, 3 diploid non-hybrids) of the D. pulex complex (see Additional file 1) were amplified using Pok5026F and the primer 28SR (5'TCCATTCGTGCGCGTCACTAATTAGATGAC), which is located 46 bp downstream of the Pokey TTAA target site. PCR reactions were performed using the Phusion ${ }^{\text {TM }}$ high-fidelity PCR kit (Finnzymes, Woburn, MA, USA) according to the supplier's protocol. The thermocycling profile consisted of 1 cycle of 1 minute at $94^{\circ} \mathrm{C}, 35$ cycles of 30 seconds at $94^{\circ} \mathrm{C}, 30$ seconds at $55^{\circ} \mathrm{C}$ and 2 minutes at $72^{\circ} \mathrm{C}$, with a final incubation of 5 minutes at $72^{\circ} \mathrm{C}$. PCR amplicons were cloned using the StrataClone ${ }^{\mathrm{TM}}$ Blunt PCR Cloning Kit (Agilent Technologies Inc., Santa Clara, CA, USA) according to the supplier's protocol. For each isolate, 10 to 12 plasmids chosen randomly from a minimum of 40 isolated colonies were purified using the E.Z.N.A. Plasmid Mini Kit II (Omega Bio-tek Inc., Norcross, GA, USA). Pokey sequences obtained from these plasmids were named using the labels of the isolate from which they were cloned plus the number that corresponds to the colony from which they were picked (for example PX2-QC-8_28 is the Pokey allele isolated from colony 28 produced during the cloning of PX2-QC-8 Pokey sequences). These Pokey alleles were compared to Pokey sequences from a previous study which focused on non-hybrid isolates [7] to ensure that the elements analyzed in this study belong to the Pokey lineage. Partial Pokey sequences were manually aligned using the ClustalW module of BioEdit v.7.0.5 [45]; available at http://www.mbio.ncsu.edu/BioEdit/bioedit.html.

\section{Detecting putative recombination events}

Potential recombination events among Pokey alleles were explored in a dataset of 53 sequences in which ambiguous sites and indels were deleted to prevent identification of false recombination events. The phi-test [46], implemented in Splitstree v4.10 [47], and GARD (Genetic Algorithm for Recombination Detection, available via the DataMonkey website at http://www.data monkey.org [48]) were used to determine if recombination events may be detected in our Pokey sequence dataset as well as in partial transposase sequences included in them.

Hudson and Kaplan minimum recombination events, $R_{m}$ [49] were calculated with the DnaSP v5 software [50]. Moreover we used kwarg (available at http://www. stats.ox.ac.uk/ lyngsoe/section26/), a heuristic alternative to the branch and bound method implemented in beagle [51], to calculate $R_{h}$ [52], which gives an estimate of the lower bound of the minimum number of recombination events. Kwarg does not guarantee that the minimal recombination history will be found but returns a history with a low number of recombination events under the infinite sites assumption. We ran kwarg 2,048 times with the default scoring scheme and recorded the number of recombination events for each iteration. 


\section{Recombination rate parameter}

As all the recombination analyses detected recombi nation events (see Results), we estimated Pokey's population recombination rate parameters along the sequence using a full likelihood coalescent approach based on a finite-sites model implemented in LAMARC v2.1.6 [53]. This approach estimates the parameter $r_{L A M}=c / \mu$ where $c$ is the recombination rate per site per generation and $\mu$ is the neutral mutation rate per site per generation. This model considers that (a) all recombination is homologous, (b) there is no association between recombination frequency and sequence divergence, (c) gene convergence and interference did not occur, (d) recombination events are selectively neutral and (e) recombination rate does not vary along the sequence. The F84 evolutionary model was used as it is a closer fit to the substitution model, Tamura 3-parameter with gamma shape and invariant sites, inferred with MEGA5 [54] than the other model (GTR) offered by LAMARC. The F84 model is also computationally faster. To account for substitution rate differences between nonsynonymous sites and all other sites, two categories of relative mutation rate were assigned. Our sampling strategy included 20 initial chains of 20,000 genealogies and two final chains of 1,000,000 genealogies with 2,500 samples discarded per chain. Adaptive heating was used to improve the search of parameter space with initial relative temperature of $1,1.1$ and 2 . The entire analysis was replicated four times and the results were combined using the algorithm of Geyer [55]. Coalescent-based analyses in LAMARC estimate both genetic diversity at the population level, $\theta_{L A M}=4 N_{e} \mu$ (where $\mu$ is the mutation rate per site for a diploid population and $N_{\mathrm{e}}$ is the effective population size) and the recombination rate $r_{L A M}=c / \mu$ (where $c$ is the rate of recombination per site per generation). Therefore, the population recombination rate parameter can be calculated as $R=\theta_{L A M} r_{L A M}=$ $\left(4 N_{e} \mu\right)(\mathrm{c} / \mu)=4 N_{\mathrm{e}} c$.

\section{Detection of recombination breakpoints}

Nucleotide diversity among sequences (that is, $\pi$ and $\theta$ ) and mutation rate heterogeneity along sequences (the Gamma shape parameter, $\alpha$ ) may have a dramatic impact on the performance of different methods to detect recombination breakpoints [56-58]. For example, Posada and Crandall [57] have shown that different methods may have a different propensity to detect recombination when nucleotide diversity varies and mutation rate heterogeneity is fixed. Moreover, they have shown that mutation rate heterogeneity may lead some methods to detect false positives [56-58].

We estimated nucleotide diversity ( $\pi$ and $\theta$ ) using DnaSP v5.10.01 [50]. Mutation rate heterogeneity $(\alpha)$ and a nucleotide substitution model were inferred using a maximum likelihood framework in MEGA5 [54]. To detect recombination breakpoints and identify which parental sequences formed the recombinant alleles, we applied a stepwise protocol of the maximum chi-square method $[59,60]$ using the stepwise package v0.1.1 (available at http://stat.sfu.ca/statgen/research/stepwise.html). The maximum chi-square method is a sliding window approach that computes a chi-square statistic from a $2 \mathrm{x}$ 2 table with counts of matches and mismatches between the two half windows separated by a proposed breakpoint. As high mutation rate heterogeneity in Pokey sequences (see Results) may lead to false negatives when window half-widths are too small, we used different values of window half-width $(70,80,90$ and 100 nucleotides) and 100,000 Monte Carlo replicates for the permutation distribution. The maximum chi-square analysis was reiterated including breakpoints detected by previous steps, as the stepwise protocol specifies, until no further breakpoint was detected. This maximum chi-square analysis identifies two sequences involved (one derived, one parental) for each recombination event detected. Recombination breakpoint detection by the maximum chisquare method is relatively more powerful and less prone to type I error (false positives) than other algorithms $[56,57]$ when sequences are subject to high mutation rate heterogeneity. Each event detected between different sequence pairs was checked by eye.

\section{Phylogenetic analyses and ancestral recombination graphs}

Dendrograms were produced for each fragment separated by recombination breakpoints highlighted by GARD analysis of the 53 sequences using the Neighbor-joining algorithm [61]. We constructed a phylogenetic network using the NeighborNet algorithm implemented in Splitstree v4.10 [47]. To understand more accurately the process and evolutionary history of recombination in the Pokey fragments cloned here, we constructed an ancestral recombination graph using kwarg $[51,52,62]$. We chose the simulation with the lowest $R_{h}$ value to build this graph.

\section{RFLP analysis}

Given the intra- and intergenomic variability of Pokey alleles in isolates from which elements were cloned, and the high number of recombination events in these sequences (see Results), we aimed to explore the presence/absence of these alleles in additional isolates belonging to the $D$. pulex complex. To do this, we developed a restriction enzyme analysis (that is, RFLP) based on Pokey sequences analyzed so far and the recombination breakpoints detected by the GARD analysis. We then screened Pokey alleles from 41 isolates (see Results) for RFLP haplotypes. PCR reactions (two replicates for each isolate) were performed in $25 \mu \mathrm{L}$ reaction mixtures 
containing $1 \mathrm{x}$ Econotaq PCR buffer, $2 \mathrm{mM} \mathrm{MgCl}_{2}$, $50 \mu \mathrm{M}$ dNTP, $0.1 \mu \mathrm{M}$ of each primer (Pok5026F and 28SR), and 1 unit of EconoTaq polymerase (Lucigen Corporation, Middleton, WI, USA) or using the Phusion ${ }^{\mathrm{TM}}$ high-fidelity PCR kit according to the manufacturer's protocol (Finnzymes). PCR amplifications were performed using the thermocycling profile described above. Restriction enzymes that cut at specific sites in some Pokey alleles may be used to highlight Pokey sequences that have undergone recombination events. We used NebCutter v2.0 (http://tools.neb.com/NEBcutter2/) on each of the 53 sequences to choose enzymes that would highlight different pure and recombinant Pokey alleles. The restriction enzymes DraI, BspHI and BstEII (New England BioLabs Inc., Ipswich, MA, USA) were used in a two-step protocol. DraI, BspHI and BstEII enzymes cut at $380 \mathrm{bp}$, approximately $790 \mathrm{bp}$, and approximately $1080 \mathrm{bp}$. The eight possible conformations were differentiated on a $3 \%$ agarose gel. In the first step, DraI and BspHI were used in the same mix containing NEBbuffer4 and digestion was conducted at $37^{\circ} \mathrm{C}$ for two hours. In the second step, NEBbuffer 3 at a $1 \mathrm{X}$ final concentration, BSA at $1 \mathrm{U} / \mu \mathrm{l}$ and BstEII at $1 \mathrm{U} / \mu \mathrm{l}$ were added to the solution and digestion was conducted at $60^{\circ} \mathrm{C}$ for one hour. Digested PCR products were separated on $3 \%$ agarose gels for two hours at 95 volts.

Cloning and PCR amplifications may produce chimeric sequences (for example, recombinants between alleles) and lead to an overestimation of recombination in nature. We assessed this hypothesis by re-amplifying Pokey sequences from isolates used in our phylogenetic survey using the Phusion ${ }^{\mathrm{TM}}$ high-fidelity PCR kit and by increasing elongation steps of the thermocycling profile to three minutes instead of two minutes. Increasing the elongation steps should reduce the probability of producing chimeric sequences [63]. Results were compared to PCR-RFLP results with two-minute elongation steps to determine if the recombination pattern is reduced. Moreover, if recombination events identified in the data are not artifacts, PCR-RFLP results should be concordant with expectations based on the cloned elements that we sequenced.

\section{Results}

\section{Structure of the Pokey transposase gene}

Sequencing of RT-PCR products, performed on RNA extracted from a sexual isolate of D. pulex (PX2-ON-9, Additional file 1), showed that a sequence upstream of the proposed stop codon in the $6.6 \mathrm{~kb}$ Pokey element is actually the beginning of a 68 bp canonical GT-AG intron. When spliced out, this adds another exon (exon 2) and extends the transposase coding region by 585 bp (Figure 1).

\section{Recombination signals}

Forty-four sequences of the 3' end of Pokey elements were added to nine Pokey alleles previously sequenced from non-hybrid isolates for a total of 53 sequences analyzed. These sequences have variable lengths due to multiple indels and vary from 1,419 bp (PX2-QC-8_30) to 1,471 bp (TE3-MB-4_9). These sequences contain two exons of the Pokey transposase gene separated by an intron of $68 \mathrm{bp}$ to $70 \mathrm{bp}$. Only the last $259 \mathrm{bp}$ of exon 1 is included, but exon 2 is complete and varies in length from $585 \mathrm{bp}$ to $627 \mathrm{bp}$. Nucleotide diversity estimates based on the full-length Pokey sequences are $\pi=0.048$ and $\theta=0.037$, and the gamma shape parameter $(\alpha)$ is 0.8423 with $63.3 \%$ of sites being invariant. Nucleotide diversity in exon 2 is an order of magnitude higher than exon 1 and about twice as high as diversity in the noncoding region downstream of the transposase gene (Table 1). Total amino acid (aa) sequences encoded by the two exons range from 282 aa in most sequences to 297 aa with most of the length variation (196 aa to 210 aa) occurring in exon 2. One sequence (TE3-MB-1_14) has a one base pair insertion that causes a frameshift mutation in the first exon.

Recombination analyses performed on the 53 Pokey sequences, as well as in the reduced dataset (about 852 bp to $891 \mathrm{bp}$ in length) of 53 partial transposase sequences, showed evidence of recombination events (phi-test; $P<0.01$ ). Two recombination breakpoints were found using GARD analysis of the entire sequences (at bp 540 and bp 820; Kishino-Hasegawa-tests, $P<0.001$ for each breakpoint) and in the transposase coding region dataset (at bp 498 and bp 686; KishinoHasegawa-tests, $P<0.001$ for each breakpoint). Six of the seven recombination events detected by the chi-square method are encountered in exon 2 (see Additional file 2 ). One step, using the stepwise protocol of the chisquare maximum method with the different half width windows (70, 80, 90 and 100), was sufficient to detect at least 20 recombination events, named $A$ to $T$ (see Additional file 3). The intricate way in which the numerous recombination events shape the evolution of Pokey elements led us to identify groups of sequences that we named $\mathbf{a}, \mathbf{b}, \mathbf{c}, \mathbf{d}, \mathbf{e}, \mathbf{f}, \mathbf{g}, \mathbf{h}$ and $\mathbf{i}$ (see Additional file 4). The phylogenetic network generated from 53 partial Pokey sequences shows clear groups of sequences (Figure 2 and Additional file 4). Six sequences (TE3MB-4_9, PX2-QC-8_1, PX2-QC-8_29, PX3-QC-1_20, EPC2-SP-2_1 and EPC-DE-3_1) were not included in groups as they may have originated from different recombination events than did the sequences with which they cluster in the network (Table 2 and Figure 2). Sequences amplified from some isolates belonging to the same species clustered together, each in a distinct group (for example, D. tenebrosa group d, European D. pulex 


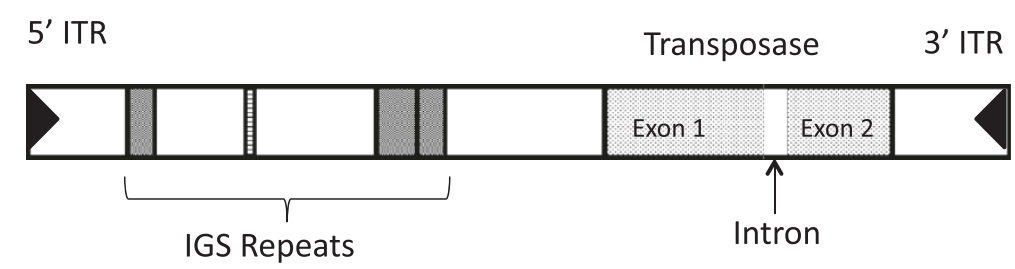

Figure 1 Schematic view of a Daphnia Pokey element. IGS repeats (gray boxes) are sequences similar to a region of the D. pulex intergenic spacer. 5'- and 3'-ITR are imperfect 16 bp inverted terminal repeats. IGS, intergenic spacer.

group e, D. pulex group h, Figure 2). Sequences from the isolate TE3-MB-4 (a + TE3-MB-4_9) and sequences from triploid isolates from Churchill (PC3-MB-6 and MI3-MB-2, group c), which have D. pulicaria or D. middendorffiana sensu stricto mtDNA haplotypes [35], also form separate groups. Various sequences amplified from D. pulicaria (PC2-QC-4 and PC2-SK-5) or hybrid isolates (PX2-QC-8, PX3-QC-1 and PC3-QC-1) clustered together in group g. Three groups included sequences amplified from isolates that belong to different species (Figure 2). Two groups ('b + EPC2-SP-2_1 + EPCDE-3_1' and f) included sequences from pulex-pulicaria hybrid isolates (PX2-QC-8 and PC3-QC-3), D. tenebrosa isolates (TE3-MB and TE2-MB) and European D. pulicaria isolates (EPC2-SP-2 and EPC-DE-3). Group $\mathbf{i}$ is represented by sequences from $D$. arenata (AR2-OR-1), D. pulicaria (PC2-QC-4) and a pulex-pulicaria hybrid (PX2-QC-8). An allele from one D. tenebrosa isolate (TE2-MB-2) grouped with sequences from pulexpulicaria hybrid isolates in group $\mathbf{g}$.

Groups of sequences with the same recombination site may represent a single recombination event between ancestral sequences. For example, the recombination breakpoint of events A (a : c), B (a : h), C (a : PX2-QC8_29), D (j : TE3-MB-4_9) and E (i : a) were estimated to be bp 592 to bp 607 for all pairs. Thus, we considered them to be the same recombination event, I. This approach results in seven different recombination events named I (bp 592 to bp 607), II (bp 748 to bp 800), III (bp 815 to bp 856), IV (bp 825 to bp 856), V (bp 917 to bp 920), VI (bp 952 to bp 957) and VII (bp 1,038 to bp 1,073) (see Additional file 4). Although it is still possible, it seems unlikely that all recombination events between

Table 1 Nucleotide diversity and mean similarity between sequence pairs for each part of the Pokey sequences

\begin{tabular}{|c|c|c|c|c|c|c|}
\hline & \multicolumn{3}{|c|}{ Coding } & \multicolumn{3}{|c|}{ Non-coding } \\
\hline & Total & exon 1 & exon 2 & Total & intron & $3^{\prime} \mathrm{NCR}$ \\
\hline$\pi$ & 0.055 & 0.007 & 0.076 & 0.038 & 0.038 & 0.038 \\
\hline$\theta$ & 0.037 & 0.009 & 0.049 & 0.036 & 0.033 & 0.036 \\
\hline Mean (\%) & 94.2 & 99.3 & 93.1 & 96.0 & 96.0 & 96.0 \\
\hline
\end{tabular}

$3^{\prime} \mathrm{NCR}$, non-coding region downstream of the transposase gene. sequence pairs were independent and not due to past events in the common ancestors of groups of sequences.

The Hudson-Kaplan minimum number of recombination events $\left(\mathrm{R}_{\mathrm{m}}\right)$ in our partial Pokey sequences dataset is 30 according to the algorithm implemented in DnaSP v5.10.01. According to the kwarg algorithm, $R_{h}$, the estimated lower bound of the minimum number of recombination events, follows a normal distribution with mean 96.39 and a standard deviation of 3.10.

\section{Recombination rate}

Using LAMARC to co-estimate mutation rate $\theta_{L A M}=$ $5.94 \times 10^{-2}$ (with $95 \%$ support intervals of $3.63 \times 10^{-2}$ and $8.16 \times 10^{-2}$ ) and the overall recombination rate $r_{L A M}=$ $5.09 \times 10^{-1}$ (with $95 \%$ support intervals of $3.57 \times 10^{-1}$ and $7.68 \times 10^{-1}$ ) allowed us to estimate a population recombination rate of $3.02 \times 10^{-2}$ recombination events per site per generation for Pokey elements.

\section{Ancestry of recombinant Pokey sequences}

Recombination breakpoints estimated by the GARD analysis (at bp 540 and bp 820) seem to correspond to the recombination breakpoints of events I and IV estimated using the maximum chi-square analysis (at bp 592 to bp 607 and bp 825 to bp 856, respectively). Dendrograms (Figure 3) were produced from each fragment (from bp 1 to bp 540, from bp 541 to bp 820, and from bp 821 to bp 1,450, respectively) of the Pokey sequences bound by recombination breakpoints identified by the GARD analysis. Combining the dendrogram analysis (Figure 3) and the maximum chi-square method of recombination breakpoint detection (see Additional file 3) allowed us to estimate from which parental sequences the recombinant Pokey sequences originated. Recombination events and probable parental sequences of each group of sequences are summarized in Table 2. While groups $\mathbf{d}$, e and $\mathbf{f}$ do not seem to derive from any recombination event (Figure 3, Table 2), other sequences may have originated from multiple events (TE3-MB-4_9 and PX3QC-1_20; Table 2). Moreover, some sequences may have originated from recombination events between sequences that were themselves recombinant. For example, sequences from group a originated from recombination between sequences from groups $\mathbf{h}$ or $\mathbf{i}$ and 


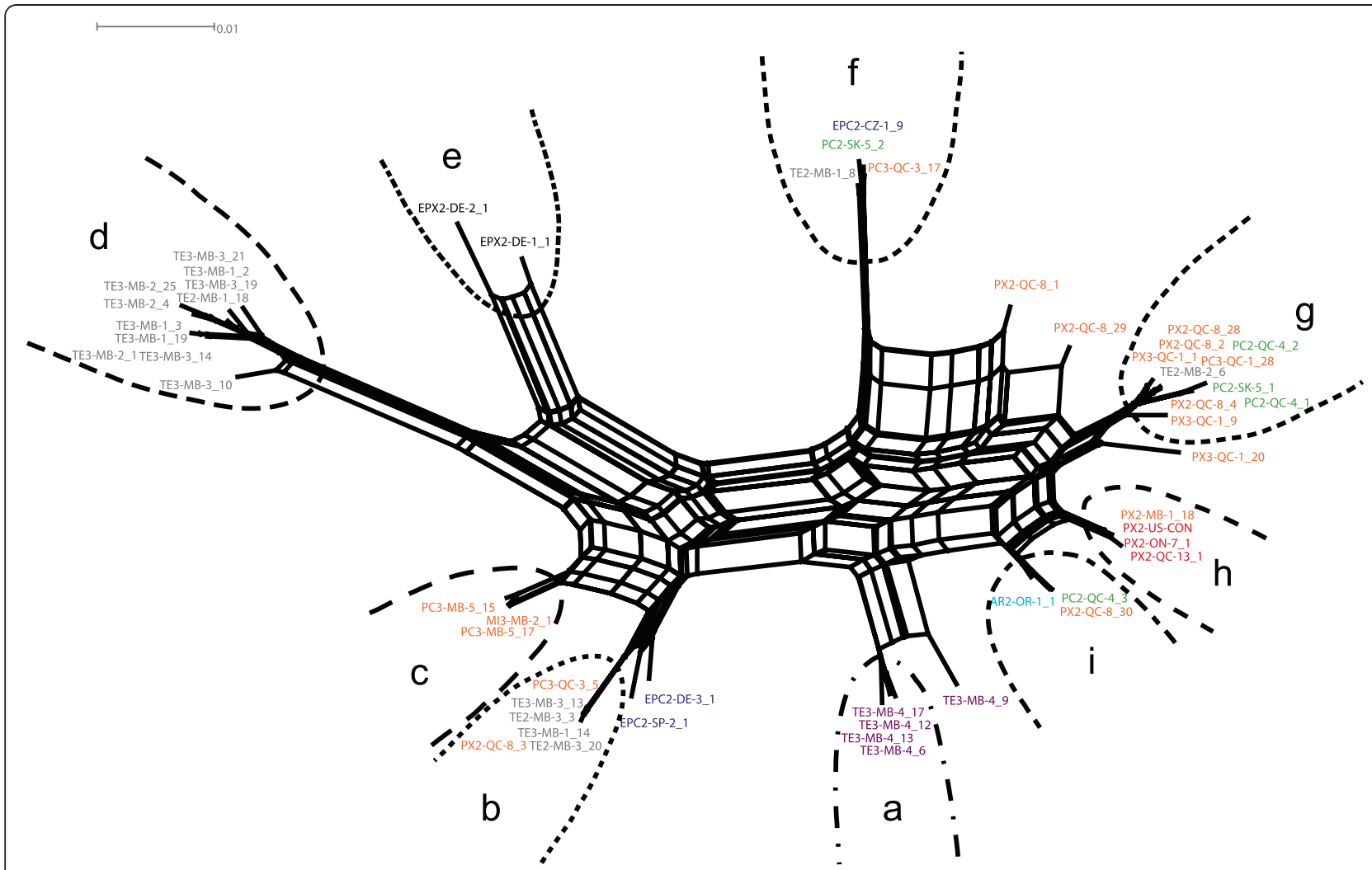

Figure 2 Phylogenetic network of 53 partial Pokey sequences from isolates of the Daphnia pulex complex. The network was constructed using the NeighborNet algorithm. Dashed lines delineate sequence groups presented in Table 2. Colors represent species or hybrid state of isolates: red $=D$. pulex, light blue $=D$. arenata, grey $=D$. tenebrosa, black $=$ European $D$. pulex, dark blue $=$ European $D$. pulicaria, green $=D$. pulicaria, orange $=$ putative pulex-pulicaria hybrids, purple $=$ the isolate with $D$. tenebrosa $\mathrm{mtDNA}$ and a $D$. pulex nuclear genome.

from group c. This last group may originate from a recombination event between sequences from groups d and $\mathbf{i}$ (Table 2, Figure 3).

Non-synonymous polymorphism at the nucleotide level leads to a high level of polymorphism at the aa level and, coupled with recombination events, produces a diversity of partial aa sequences of the Pokey transposase (see Additional file 2).

\section{Ancestral recombination graphs}

Additional file 5 shows an ancestral recombination graph with 89 recombination events $\left(R_{h}\right)$ identified by the Song and Hein algorithm [52]. The evolutionary history of Pokey allele TE3-MB-4_12, which contains 38 recombination events, is highlighted in red. This ancestral recombination graph shows putative past recombination events that were not detected by the maximum chi-square method. For example, the evolution of EPX2-DE-1_1, encountered in a European D. pulex isolate, seems to involve 25 past recombination events.

\section{Pokey alleles in the v1.1 Daphnia genome}

The v1.1 genome sequence of D. pulex [36] was scanned for our partial Pokey sequence using TBLASTN to explore which alleles are present. Sequences from contigs with $\mathrm{E}$-value $=0.0$ and without indels more than 10 nucleotides long were aligned with our 53 sequences to generate a dataset of 58 sequences. A NeighborNet phylogenetic network (see Additional file 6) shows that Pokey elements from the genome sequence group with sequences encountered in diploid and polyploid isolates from the pulicaria group (PC2-SK-5 and PC3-QC-3), in diploid D. tenebrosa (TE2-MB-1) and in European $D$. pulicaria (EPC2-CZ-1).

\section{RFLP analysis of Pokey alleles in additional isolates}

To test if recombinant Pokey alleles are encountered in additional clones of the $D$. pulex complex, we used a restriction enzyme analysis (that is, PCR-RFLP) of alleles amplified from the 28S rRNA genes from 41 isolates (see Additional file 7). Eight different haplotypes were found (numbered 1 to 8) using three restriction enzymes, some of which correspond to sequences from 


\begin{tabular}{|c|c|c|}
\hline Sequences & $\begin{array}{l}\text { Events involving sequences according to the } \\
\text { maximum } \chi^{2} \text { test (other sequences involved }{ }^{\mathrm{a}} \text { ) }\end{array}$ & $\begin{array}{l}\text { Pairs of probable parental sequences estimated using GARD } \\
\text { dendrograms based on two different recombination breakpoints }\end{array}$ \\
\hline a & $\begin{array}{l}\text { I (h, g, c, PX2-QC-8_29), IV (b, EPC2-SP-2_1), V (h), VII } \\
(\mathbf{g})\end{array}$ & $\mathbf{h}: \mathbf{c}$ or $\mathbf{i}: \mathbf{c}$ \\
\hline $\begin{array}{l}\text { TE3-MB- } \\
4 \_9\end{array}$ & I (i), VII (g, PX2-SF-8_29, PX2-SF-8_1, f) & $\mathbf{h}: \mathbf{c}$ or $\mathbf{i}: \mathbf{c}$ and $\mathbf{c}: \mathbf{h}$ \\
\hline \multirow[t]{3}{*}{ b } & II (i), IV (a) & $\mathbf{d}: \mathbf{a}$ or EPC2-SP-2_1 $: \mathbf{a}$ or EPC2-DE-3_1 : a \\
\hline & & $\mathbf{d}: \mathbf{c}$ or EPC2-SP-2_1 : c or EPC2-DE-3_1 : c \\
\hline & & $\mathbf{d}: \mathbf{i}$ or EPC2-SP-2_1 $: \mathbf{i}$ or EPC2-DE-3_1 $: \mathbf{i}$ \\
\hline $\begin{array}{l}\text { EPC2-SP- } \\
2 \_1\end{array}$ & II (i), IV (a, h) & $\mathbf{b}: \mathbf{i}, \mathbf{b}: \mathbf{c}$ or $\mathbf{b}: \mathbf{a}$ \\
\hline $\begin{array}{l}\text { EPC2-DE- } \\
3 \_1\end{array}$ & - & $\mathbf{b}: \mathbf{i}, \mathbf{b}: \mathbf{c}$ or $\mathbf{b}: \mathbf{a}$ \\
\hline c & $\mathbf{I}(\mathbf{a}), \mathbf{I V}(\mathbf{i}), \mathbf{V}(\mathbf{h}), \mathbf{V I}(\mathbf{g})$ & d : i \\
\hline d & - & - \\
\hline e & - & - \\
\hline f & VII (TE3-MB-4_9) & - \\
\hline $\begin{array}{l}\mathrm{PX2-QC-} \\
8 \_29\end{array}$ & I (a), VII (TE3-MB-4_9) & $i: f$ or $h: f$ \\
\hline $\begin{array}{l}\mathrm{PX} 2-\mathrm{QC}- \\
8 \_1\end{array}$ & VII (TE3-MB-4_9) & $g: f$ \\
\hline g & I (a), VI (c), VII (a, TE3-MB-4_9) & unknown : $f$ \\
\hline $\begin{array}{l}\mathrm{PX3}-\mathrm{QC}- \\
1 \_20\end{array}$ & III (i) & $\mathbf{g}: \mathbf{h}$ or $\mathbf{g}: \mathbf{i}$ and $\mathbf{i}: \mathbf{g}$ or $\mathbf{h}: \mathbf{g}$ \\
\hline $\mathrm{h}$ & IV (EPC2-SP-2_1), V (a, c) & i : Unknown or a : Unknown \\
\hline i & I (TE3-MB-4_9), II (b, EPC2-SP-2_1), III (PX3-QC-1_20), IV (c) & h : Unknown \\
\hline
\end{tabular}

the groups encountered in our phylogenetic analysis. The distribution of these haplotypes is summarized in Table 3. We found that rRNA genes from different genomes may contain up to five different Pokey RFLP haplotypes with an average of 1.74 per isolate and a standard deviation of 0.96 .

\section{Discussion}

\section{Hybridization among Daphnia species}

Our results show that recombination has a dramatic impact on the evolution of Pokey alleles in the rDNA of Daphnia. The recombinant sequences do not seem to be due to cloning artifacts as some isolates may have just one recombinant Pokey allele in their genome (for example, triploid isolates PC3-SK-5 and PC3-MB-4 or diploid isolates EPC2-CZ-1, EPC2-SP-2 and PX2-QC-1, Additional file 7). Increasing the extension time of the PCR cycles to avoid artefactual recombination events during amplification did not change the RFLP results (data not shown). Moreover, Daphnia isolates show RFLP patterns that are concordant with those expected from the cloned Pokey sequences (see Additional file 7).
Pokey sequences show substantial nucleotide diversity ( $\pi=0.048$ and $\theta=0.037$, Table 1 ), undergo recombination at a rate of $3.02 \times 10^{-2}$ events per site per generation and rarely spread by horizontal transfer [7]. As predicted in the case of recombination events between Pokey elements that co-evolve with their host and have undergone recent activity, hybrid isolates from the D. pulex complex carry either sequences inherited from putative parental species or mosaic sequences produced by recombination between parental types. Most Pokey sequences from pulex-pulicaria hybrids (for example, PX2-MB-1, PX3-QC-1_1, PC3-QC-1_28 or PX2-QC8_28) either cluster with Pokey alleles from D. pulex (group h) or D. pulicaria (PC2-QC-4 and PC-SK-5_2 in group g) in the phylogenetic network (Figure 2) or are recombinants between sequences from these groups (that is, PX3-QC-1_20). Pokey sequences from polyploid isolates with mtDNA from either $D$. pulicaria or $D$. middendorffiana from Manitoba, Canada seem to be recombinants between sequences found in D. tenebrosa (group d) and D. pulicaria, D. pulex or D. arenata (that is, groups $\mathbf{g}, \mathbf{h}$ or $\mathbf{i}$, respectively, Table 2 ). This suggests that D. tenebrosa may be one of the parental species of these 


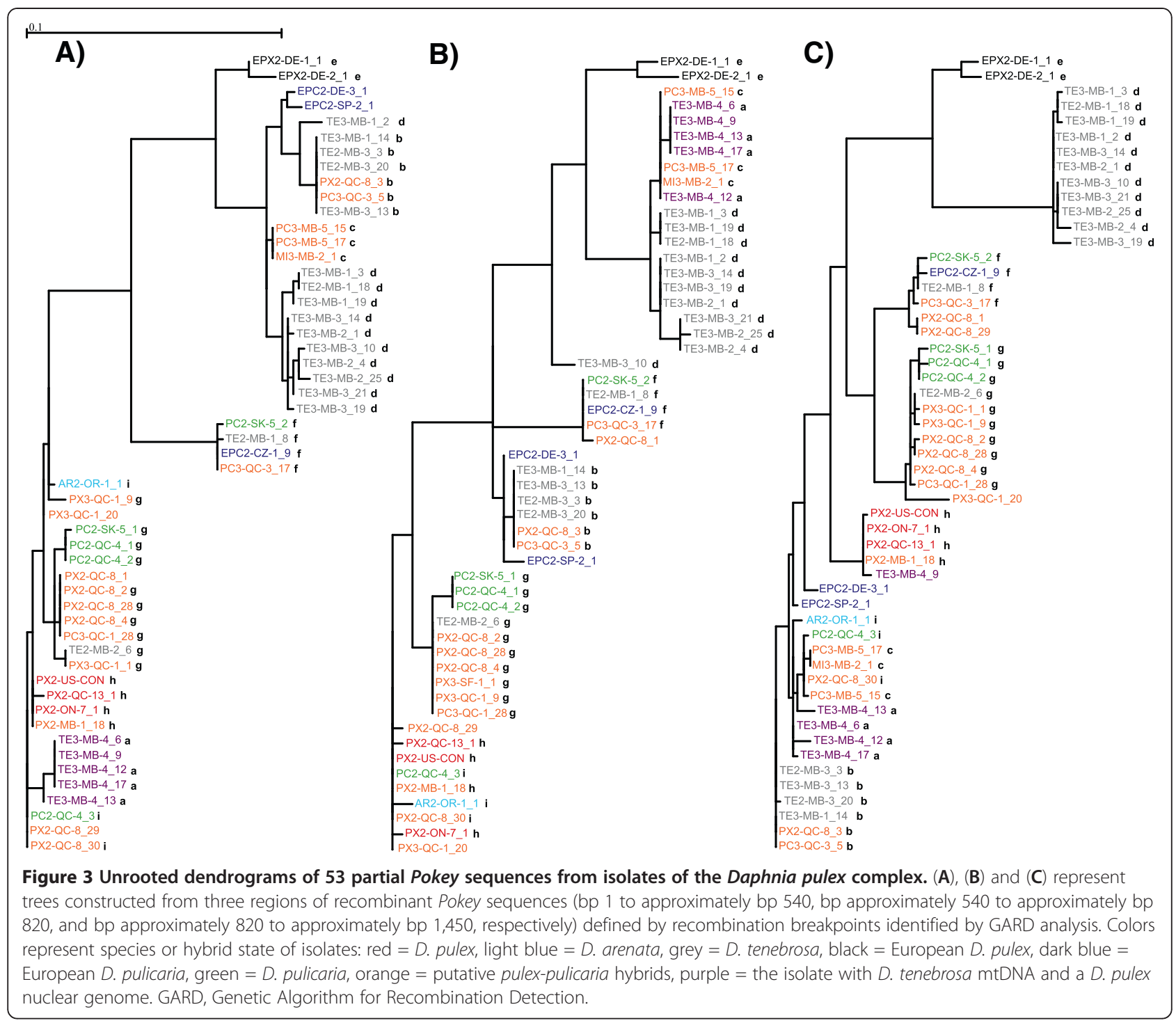

Table 3 Taxonomic, habitat and geographical distributions of RFLP haplotypes

\begin{tabular}{|c|c|c|c|}
\hline Haplotype & Percentage and taxonomic occurrence & Habitat & Geographic location \\
\hline 1 & $33.33 \%$ of isolates, mainly with D. pulex mitochondria & ponds & $\mathrm{QC}, \mathrm{ON}, \mathrm{MB}(\mathrm{CAN}), \mathrm{MI}(\mathrm{USA})$ \\
\hline 2 & $\begin{array}{l}38.09 \% \text { of isolates, D. pulicaria, polyploids with D. pulicaria mitochondria, diploid/ } \\
\text { triploids with D. pulex mitochondria and one D. tenebrosa isolate }\end{array}$ & $\begin{array}{l}\text { Various } \\
\text { habitats }\end{array}$ & various locations \\
\hline 3 & $\begin{array}{l}26.19 \% \text { of isolates, D. pulex, diploid hybrids with D. pulex mitochondria and triploid } \\
\text { hybrids D. pulicaria mitochondria, European D. pulicaria and D. tenebrosa isolâtes }\end{array}$ & $\begin{array}{l}\text { various } \\
\text { habitats }\end{array}$ & various locations \\
\hline 4 & $\begin{array}{l}30.95 \% \text { of isolates, D. pulex, diploid hybrids with } D \text {. pulex mitochondria and triploid } \\
\text { hybrids D. pulicaria mitochondria, D. middendorffiana, European D. pulicaria and D. } \\
\text { tenebrosa isolates }\end{array}$ & $\begin{array}{l}\text { various } \\
\text { habitats }\end{array}$ & various locations \\
\hline 5 & One introgressed isolate (TE3-MB-4) & $\begin{array}{l}\text { rock bluff } \\
\text { pools }\end{array}$ & Churchill MB, (CAN) \\
\hline 6 & $\begin{array}{l}9.76 \% \text { of isolates, D. pulex, D. pulicaria or D. middendorffiana and D. pulex-D. pulicaria } \\
\text { hybrids }\end{array}$ & $\begin{array}{l}\text { ponds or rock } \\
\text { bluff pools }\end{array}$ & $\begin{array}{l}\text { Kujjuarappik and Metis, QC } \\
\text { (CAN) Churchill, MB (CAN) }\end{array}$ \\
\hline 7 & One D. pulex-D. pulicaria hybrid (PX2-MI-11) & ponds & MI (USA) \\
\hline 8 & $26.8 \%$ of isolates, D. pulex and D. pulicaria & $\begin{array}{l}\text { various } \\
\text { habitats }\end{array}$ & various locations \\
\hline
\end{tabular}


polyploid isolates, which are thought to originate from hybridization between $D$. pulex and D. pulicaria or with another species that no longer exists as a cyclic parthenogen [22,29,30,32]. Phylogenetic analysis of LdhA sequences is also concordant with a hybrid origin of polyploid isolates with $D$. middendorffiana mtDNA (Figure 3 in [64]).

The introgressed triploid isolate, TE3-MB-4, which has $D$. tenebrosa mtDNA and most likely a $D$. pulex nuclear genome [22,31]), carries alleles from group 'a + TE3MB-4_9' with a minor trace from $D$. tenebrosa and major traces from $D$. pulex or D. pulicaria alleles. The presence of alleles with traces of $D$. pulex or D. pulicaria in $D$. tenebrosa or European D. pulicaria isolates (TE3-MB-1, TE3-MB-3, TE2-MB-1, TE2-MB-3, EPC2MB-2 and EPC2-MB-3) may be a remnant of hybridization events between these four species. Similarly, Pokey alleles from European D. pulicaria isolates (EPC2-SP-2_1 and EPC2-DE-3_1) also seem to be recombinants between alleles from North American $D$. pulex or D. pulicaria and D. tenebrosa (recombination events G, K and L in Table 2 and Figure 2). Although Vergilino et al. [22] did not detect evidence of hybridization between species in the pulicaria group (North American D. pulex and D. pulicaria) and the tenebrosa group (D. tenebrosa and European D. pulicaria) using microsatellite loci, Weider et al. [33] did find evidence for it using allozyme data. Moreover, Ambrose and Crease [34] have shown that IGS segments from some isolates of European D. pulex may have originated from North American D. pulicaria. As North American D. pulex and D. pulicaria have been found in Europe [21], introgression of Pokey alleles from North American species into European species is possible and may generate recombinants that persist for long periods of time and are able to spread over broad geographic areas.

Although most Pokey sequences from hybrid isolates are mosaics, which is consistent with recent recombination between alleles from different parent species, some recombinant alleles may be more ancient and represent retained ancestral polymorphism (that is, incomplete lineage sorting). The clustering of recombinant alleles in group $\mathbf{b}$, which are encountered in $D$. tenebrosa isolates, a hybrid D. pulex (PX2-QC-8) and a polyploid with $D$. pulicaria mtDNA (PC3-QC-3) suggest that these recombinant alleles are ancient.

Pokey alleles from the isolate whose genome was sequenced [36] cluster with alleles from group $\mathbf{f}$ (Figure 3 and Additional file 6) whereas Pokey alleles from other $D$. pulex isolates cluster in group $\mathbf{h}$. This may be the result of incomplete lineage sorting or a signature of past hybridization events that have occurred in the population from which the genome isolate was sampled.
Alternatively, the genomic Pokey alleles may have a different evolutionary history than their paralogs in rDNA. Moreover, these genomic alleles show a limited signature of recombination according to the GARD analysis. As the recombination rate is variable across the Daphnia genome [65,66], we may expect this result if Pokey elements are inserted in genomic regions showing low recombination rates.

\section{Recombination parameters among Pokey elements}

The number of recombination breakpoints detected in our sampled sequences by different methods was highly variable. Most recombination breakpoint detection algorithms underestimate the number of recombination events [57] and their accuracy varies depending on whether the method uses summary statistics or the maximal information in the sample [58]. The maximum chi-square method performed in a stepwise manner and for different window sizes detected a minimum of seven unique recombination events between Pokey alleles from different species of the $D$. pulex complex (see Additional file 3 , Table 2). This value is likely to be an underestimate as a high recombination rate may lead to overlapping recombination events. As the maximum chi-square is a substitution-based method that compares substitution distributions from two windows flanking a tested site, recombination breakpoint detection will be dependent on the size of the windows used when mutation rate heterogeneity is high and/or nucleotide diversity is low. Small windows should increase the probability of detecting recombination breakpoints in high diversity regions but may be unable to detect recombination events in low nucleotide diversity regions. Increasing the size of the windows to increase recombination detection accuracy in regions with low nucleotide diversity may lead to less accuracy in the regions with multiple recombination events. The estimate of Hudson-Kaplan minimum recombination events $\left(R_{m}\right)$ is 30 and the haplotype lower bound $\left(R_{h}\right)$ estimate is $96.39 \pm 3.10 . R_{m}$ is a parsimony-based method (that is, the four gamete test) and may underestimate the actual lower bound of recombination events, particularly when the recombination rate is high $[67,68]$. In contrast to the former estimators, $R_{h}$, estimated using a maximum-likelihood framework that uses the maximal information in the sample, is probably more reliable than $R_{m}[68]$ and shows the highest lower bound of recombination events. The accuracy of $R_{m}$ and $R_{h}$ to estimate the lower bound of recombination events has not been tested on sequences subjected to low and high heterogeneous mutation rates. If numerous recombination events occurred during the evolutionary history of Pokey elements, we may need sophisticated phylogenetic tools and analyses, such as the Ancestral Recombination Graph, to deepen our understanding of their evolutionary history. 
The recombination rate of Pokey sequences estimated in our survey is high $\left(3.02 \times 10^{-2}\right.$ events/site/generation) but comparable to the value $\left(2.0\right.$ to $6.0 \times 10^{-2}$ events/ generation) calculated by McTaggart et al. [65] based on changes in the relative frequency of rDNA length variants in four apomictically-propagated Daphnia obtusa lines over 90 generations. To our knowledge, this is the first time that the rate of recombination has been explicitly estimated for a DNA transposon. This estimate has to be viewed with caution as some of the assumptions of the algorithm used, such as the absence of gene conversion and interference, and the selective neutrality of recombination events, may be violated. Even so, $R_{h}$ and $\mathrm{R}_{\mathrm{m}}$ estimates lead to the conclusion that the recombination rate is high between Pokey elements. Such a high rate of recombination between Pokey elements may explain why no significant differences were found between the amount of variation in $28 \mathrm{~S}$ rRNA genes with or without Pokey insertions in cyclic and obligately asexual D. pulex isolates [69] as recombination may take place between the Pokey elements as well as the 28S rRNA genes themselves.

\section{Effects of recombination on evolution of Pokey elements}

Numerous studies have focused on the relationship between recombination rates in host genomes and $\mathrm{TE}$ distributions [70-73] or dynamics [37,41,74]. The distribution and abundance of TEs may be influenced by selective forces [75-77], the stochastic process of mutation [78] and/or the availability of insertion sites. One theoretical model based on the action of selection posits that the impact of recombination events between TEs inserted in non-homologous loci is deleterious (that is, ectopic exchange model) and, therefore, insertions will accumulate in regions of the genome with low rates of recombination [77]. However, empirical data from sequenced genomes show that the relationship between TE density and recombination rate depends on the organism and the TE class or family [4,39,71,79-82].

Recombination between TE copies of the same family has been reported before [83-87], but the significance of this for TEs in general is rarely discussed. Our survey focused on variation in an approximately 1,600 bp sequence. This dataset does not include the TIRs known to be essential for transposition or the IGS sequences upstream of the transposase gene that may influence expression of the transposase [39]. If the TIRs or transposase gene have diverged in different species and recombination events produce mosaic sequences, the transposase may not be able to recognize TIRs with which it has not co-evolved. Thus, we might expect that many recombination events between divergent TE alleles will inactivate their mosaic product. Alternatively, Schaack et al. [74] recently suggested that TEs might benefit from recombination in a similar manner to the host, by generating new variants that are able to evade sequence-specific host suppression machinery. Recombination between different insertions could bring together independent, beneficial nucleotide substitutions that may then be favored by intragenomic selection and result in an increase in copy number of the new, recombinant insertion.

Fragments of the Pokey transposase gene have different evolutionary histories. Moreover, non-synonymous polymorphism coupled with recombination has produced a substantial level of Pokey variability at the aa level (Table 1, Additional file 2). This variability is primarily seen in exon 2 , which is thought to contain a nuclear localization signal and a sequence similar to a Plant Homeo Domain or cysteine-rich zinc finger that is possibly involved in chromatin or protein-protein interactions $[40,88,89]$. For example, the fourth cysteine residue of the putative zinc finger is replaced either by a serine or a tyrosine residue in 3 of the 40 different aa sequences we analyzed (see Additional file 2). This variation might translate to flexibility in target site interactions or changes to dimerization dynamics during the formation of the transposition complex, which could have implications for mobility and transposition efficiency.

DNA transposons in Caenorhabditis elegans are located preferentially in recombination hotspot regions whereas retrotransposons are not, and Duret et al. [71] suggested a role for recombination in the transposition process. Glass et al. [69] did find indirect evidence that Pokey insertions in rDNA could spread through unequal crossing-over, so perhaps there is some credence to this suggestion. In addition to Pokey in Daphnia, the retrotransposons $R 1$ and $R 2$ in Drosophila and numerous other arthropods are also found inserted into rDNA. Using the ectopic exchange model as a framework, Zhang et al. [90] demonstrated that eukaryotic hosts may tolerate a high load of retrotransposable elements in their rDNA because they generally have many more rDNA copies than the minimum required for rRNA synthesis. The location of Pokey elements in rDNA may be advantageous for both element and host, as frequent recombination between different copies located in these loci may increase the efficiency of intragenomic selection, and insertions may be only mildly harmful due to the multi-copy nature of rDNA. Pokey haplotype 2 is a recombinant allele (Figures 1, Table 2) that was found in numerous hybrid and non-hybrid Daphnia isolates having different origins and occupying different locations and ecosystems (Table 3). This might be such a successful haplotype due to the amalgamation, through recombination, of separately beneficial mutations into a single lineage that was then able to spread to different populations and species within the $D$. pulex complex. 


\section{Conclusions}

Most of the hybrid isolates from the D. pulex complex analyzed in this study carry Pokey sequences inherited from putative parental species or mosaic sequences produced by recombination between parental types. In addition, recombination may play an important role in generating life history variation in this TE. Future studies should test the activity of recombinant Pokey alleles to assess if recombination events have had a significant effect on their transposition capacity. Additionally, testing for recombinants among the other families of TEs found within $D$. pulex would be useful to determine whether rates of recombination among TEs depend in part on the region of the genome they inhabit or on other properties such as mode of transposition. Moreover, the possible beneficial effects of recombination on TE-level evolution in general should be investigated more thoroughly by estimating rates of recombination for different TEs in various genomes.

\section{Additional files}

Additional file 1: Description of Daphnia isolates included in this study. The labels of the isolates are composites of their characteristics. The first two letters represent the mitochondrial haplotypes $(A R=D$. arenata, $\mathrm{EPC}=$ European $D$. pulicaria, $\mathrm{EPX}=$ European $D$. pulex, $\mathrm{Ml}=\mathrm{D}$ middendorffiana, $\mathrm{PC}=\mathrm{D}$. pulicaria, $\mathrm{PX}=\mathrm{D}$. pulex, $\mathrm{TE}=\mathrm{D}$. tenebrosa) followed by the ploidy level (2 or 3), a 2 letter country or state/province code and the isolate number. Sequences marked with an asterisk $(*)$ were obtained from a RT-PCR product. Accession numbers refer to Pokey sequences amplified from Daphnia isolates.

Additional file 2: Amino acid polymorphism in $\mathbf{4 0}$ unique partial Pokey transposase sequences. The sequences were derived from 53 Pokey elements obtained from members of the Daphnia pulex complex. Numbers at each position represent the number of sequences that carry this amino acid. Not all values sum to 40 as indels occurred in some sequences. The pink bar represents the amino sequence coded by the partial exon 1. The blue bar represents the amino sequence coded by exon 2. The dark blue regions with greek numbers represent estimated regions of recombination identified using maximum chi-square analysis (Additional file 3).

Additional file 3: Recombination events in $\mathbf{5 3}$ partial Pokey sequences. Recombination events were estimated using the maximum chi-square method.

Additional file 4: Groups of recombinant Pokey sequences. The groups are based on phylogenetic network analysis (Figure 2) and recombination breakpoint analyses (Table 2).

Additional file 5: One possible ancestral Pokey recombination graph. The graph was constructed from 40 different Pokey sequences from members of the Daphnia pulex complex and shows 89 recombination events. Ovals at the bottom of the graph represent Pokey alleles. Ovals with a trifurcation represent putative recombination events. Colors represent species or hybrid state of isolates: red $=D$. pulex, light blue $=D$. arenata, grey $=D$. tenebrosa, black $=$ European $D$. pulex, dark blue $=$ European $D$. pulicaria, green $=D$. pulicaria, orange $=$ putative pulex-pulicaria hybrids, purple $=$ the isolate with $D$. tenebrosa mtDNA and a D. pulex nuclear genome. Red lines represent the evolutionary history of the Pokey allele TE3-MB-4_12.

Additional file 6: Phylogenetic network of 58 partial Pokey sequences. The network was constructed using the NeighborNet algorithm. Sequences named CO_Scaffold_Xxx were obtained from the v1.1 genome sequence of Daphnia pulex [36]. Colors represent species or hybrid state of isolates: red $=D$. pulex, light blue $=D$. arenata, grey $=D$. tenebrosa, black = European D. pulex, dark blue $=$ European D. pulicaria, green $=D$. pulicaria, orange $=$ putative pulex-pulicaria hybrids, purple $=$ the isolate with $D$. tenebrosa mtDNA and a D. pulex nuclear genome.

Additional file 7: Pokey RFLP haplotypes amplified from 41 isolates of the Daphnia pulex complex. Location codes are defined in Additional file 1. A one letter code allows differentiation of geographical regions in the same state or province: $\mathrm{C}=$ Churchill, $\mathrm{MB}, \mathrm{CAN} ; \mathrm{W}=$ Winnipeg, $\mathrm{MB}, \mathrm{CAN} ; \mathrm{K}$ $=$ Kuujjuarapik, QC, CAN; M = Metis, QC, CAN; S = Sainte-Foy, QC, CAN. Taxonomic codes are as follows: $E P C=$ European $D$. pulicaria; $P C=D$. pulicaria; $\mathrm{PX}=\mathrm{D}$. pulex; $\mathrm{TE}=\mathrm{D}$. tenebrosa; $\mathrm{Hyb}=$ pulex-pulicaria hybrid; Int = introgressed isolate.

\section{Abbreviations}

Aa: amino acid; Bp: base pair; BSA: bovine serum albumin; GARD: Genetic Algorithm for Recombination Detection; IGS: intergenic spacer; mtDNA: mitochondrial DNA; PCR: polymerase chain reaction; RFLP: restriction fragment length polymorphism; SINEs: short interspersed elements;

TEs: transposable elements; TIRs: terminal inverted repeats.

\section{Competing interests}

The authors declare that they have no competing interests.

\section{Authors' contributions}

RV designed the project and wrote the manuscript. RV and TAE planned the analyses. RV, TAE and PDP conducted the analyses. All authors analyzed the data and contributed to the writing and editing of the manuscript. All authors approved the final manuscript.

\section{Acknowledgments}

RV acknowledges a scholarship from Centre d'études Nordiques and a travel fellowship from UQAR. PDP is supported by a research grant from the Canada Research Chair program. This work was supported by Discovery Grants from the Natural Sciences and Engineering Research Council (NSERC) of Canada to FD and to TJC. We thank Yves Bigot for pointing out the existence of the intron in the Pokey transposase gene.

\section{Author details}

'Département de Biologie, Université du Québec à Rimouski, 300, allée des Ursulines, Rimouski, Québec G5L 3A1, Canada. ${ }^{2}$ Centre d'Études Nordiques, Université Laval, Pavillon Abitibi-Price, 2405, rue de la Terrasse, Local 1202, Québec GIV 0A6, Canada. ${ }^{3}$ Current address: Great Lake Institute of Environmental Research, University of Windsor, 401 Sunset Avenue, Windsor N9B 3P4, Canada. ${ }^{4}$ Department of Integrative Biology, University of Guelph, Science complex, Guelph, ON N1G 2 W1, Canada. ${ }^{5}$ College of Engineering, University of Illinois at Chicago, (M/C 159), 851 South Morgan, Chicago, IL 60607, USA.

Received: 20 June 2012 Accepted: 6 November 2012 Published: 6 February 2013

\section{References}

1. Feschotte C, Pritham EJ: DNA transposons and the evolution of eukaryotic genomes. Annu Rev Genet 2007, 41:331-368.

2. Shigenobu S, Watanabe H, Hattori M, Sakaki Y, Ishikawa H: Genome sequence of the endocellular bacterial symbiont of aphids Buchnera sp. APS. Nature 2000, 407:81-86.

3. Pidpala OV, Yatsishina A, Lukash L: Human mobile genetic elements: structure, distribution and functional role. Cytol Genet 2008, 42:420-430.

4. Eickbush DG, Eickbush TH: Vertical transmission of the retrotransposable elements R1 and R2 during the evolution of the Drosophila melanogaster species subgroup. Genetics 1995, 139:671-684.

5. Robertson HM, Lampe DJ: Recent horizontal transfer of a mariner transposable element among and between Diptera and Neuroptera. Mol Biol Evol 1995, 12:850-862.

6. Gonzalez P, Lessios HA: Evolution of sea urchin retroviral-like (SURL) elements: evidence from 40 echinoid species. Mol Biol Evol 1999, 16:938-952. 
7. Penton EH, Crease TJ: Evolution of the transposable element Pokey in the ribosomal DNA of species in the subgenus Daphnia (Crustacea: Cladocera). Mol Biol Evol 2004, 21:1727-1739.

8. Shedlock AM, Okada N: SINE insertions: powerful tools for molecular systematics. Bioessays 2000, 22:148-160.

9. Capy $P$, Anxolabéhère $D$, Langin T: The strange phylogenies of transposable elements: are horizontal transfers the only explanation? Trends Genet 1994, 10:7-12

10. Loreto ELS, Carareto CMA, Capy P: Revisiting horizontal transfer of transposable elements in Drosophila. Heredity 2008, 100:545-554.

11. Gilbert C, Schaack S, Pace li JK, Brindley PJ, Feschotte C: A role for host-parasite interactions in the horizontal transfer of transposons across phyla. Nature 2010, 464:1347-1350

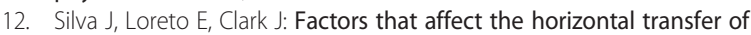
transposable elements. Curr Issues Mol Biol 2004, 6:57-71.

13. Yoshiyama M, Tu Z, Kainoh Y, Honda H, Shono T, Kimura K: Possible horizontal transfer of a transposable element from host to parasitoid. Mol Biol Evol 2001, 18:1952-1958.

14. Hebert PDN: Obligate asexuality in Daphnia. Am Nat 1981, 117:784-789.

15. Hebert PDN, Crease T: Clonal diversity in populations of Daphnia pulex reproducing by obligate parthenogenesis. Heredity 1983, 51:353-369.

16. Innes DJ, Schwartz SS, Hebert PDN: Genotypic diversity and variation in mode of reproduction among populations in the Daphnia pulex group. Heredity 1986, 57:345-355.

17. Innes DJ, Fox CJ, Winsor GL: Avoiding the cost of males in obligately asexual Daphnia pulex (Leydig). Proc R Soc Lond B Biol Sci 2000, 267:991-997.

18. Hebert $P$, Finston $T$ : Macrogeographic patterns of breeding system diversity in the Daphnia pulex group from the United States and Mexico. Heredity 2001, 87:153-161.

19. Hebert PDN: The Daphnia of North America: an illustrated fauna. Guelph, Ontario: University of Guelph; 1995.

20. Colbourne JK, Crease TJ, Weider L, Hebert PDN, Dufresne F, Hobaek A: Phylogenetics and evolution of a circumarctic species complex (Cladocera: Daphnia pulex). Biol J Linn Soc 1998, 65:347-365.

21. Marková S, Dufresne F, Rees DJ, Cerný M, Kotlík P: Cryptic intercontinental colonization in water fleas Daphnia pulicaria inferred from phylogenetic analysis of mitochondrial DNA variation. Mol Phylogenet Evol 2007, 44:42-52.

22. Vergilino R, Markova S, Ventura M, Manca M, Dufresne F: Reticulate evolution of the Daphnia pulex complex as revealed by nuclear markers. Mol Ecol 2011, 20:1191-1207.

23. Crease TJ, Stanton DJ, Hebert PDN: Polyphyletic origins of asexuality in Daphnia pulex. II. Mitochondrial-DNA variation. Evolution 1989, 43:1016-1026.

24. Hebert PDN, Beaton MJ, Schwartz SS, Stanton DJ: Polyphyletic origins of asexuality in Daphnia pulex. I. Breeding-system variation and levels of clonal diversity. Evolution 1989, 43:1004-1015.

25. Hebert PDN, Schwartz SS, Ward RD, Finston TL: Macrogeographic patterns of breeding system diversity in the Daphnia pulex group. I. Breeding systems of Canadian populations. Heredity 1993, 70:148-161.

26. Innes DJ, Hebert PDN: The origin and genetic basis of obligate parthenogenesis in Daphnia pulex. Evolution 1988, 42:1024-1035.

27. Heier CR, Dudycha JL: Ecological speciation in a cyclic parthenogen: sexual capability of experimental hybrids between Daphnia pulex and Daphnia pulicaria. Limnol Oceanogr 2009, 54:492-502.

28. Cristescu ME, Constantin A, Bock DG, Caceres CE, Crease TJ: Speciation with gene flow and the genetics of habitat transitions. Mol Ecol 2012, 21:1411-1422

29. Beaton MJ, Hebert PDN: Geographical parthenogenesis and polyploidy in Daphnia pulex. Am Nat 1988, 132:837-845.

30. Dufresne F, Hebert PDN: Hybridization and origins of polyploidy. Proc R Soc Lond B Biol Sci 1994, 258:141-146.

31. Dufresne F, Hebert PDN: Polyploidy and clonal diversity in an arctic cladoceran. Heredity 1995, 75:45-53.

32. Dufresne F, Hebert PDN: Pleistocene glaciations and polyphyletic origins of polyploidy in an arctic cladoceran. Proc R Soc Lond B Biol Sci 1997, 264:201-206.

33. Weider LJ, Hobaek A, Hebert PDN, Crease TJ: Holarctic phylogeography of an asexual species complex - II. Allozymic variation and clonal structure in Arctic Daphnia. Mol Ecol 1999, 8:1-13.
34. Ambrose $\mathrm{C}$, Crease $\mathrm{T}$ : Evolution of the nuclear ribosomal DNA intergenic spacer in four species of the Daphnia pulex complex. BMC Genet 2011, 12:13.

35. Vergilino R, Belzile C, Dufresne F: Genome size evolution and polyploidy in the Daphnia pulex complex (Cladocera: Daphniidae). Biol J Linn Soc 2009, 97:68-79.

36. Colbourne JK, Pfrender ME, Gilbert D, Thomas WK, Tucker A, Oakley TH, Tokishita S, Aerts A, Arnold GJ, Basu MK, Bauer DJ, Cáceres CE, Carmel L, Casola C, Choi JH, Detter JC, Dong Q, Dusheyko S, Eads BD, Fröhlich T, Geiler-Samerotte KA, Gerlach D, Hatcher P, Jogdeo S, Krijgsveld J, Kriventseva EV, Kültz D, Laforsch C, Lindquist E, Lopez J, et al: The ecoresponsive genome of Daphnia pulex. Science 2011, 331:555-561.

37. Schaack S, Choi E, Lynch M, Pritham E: DNA transposons and the role of recombination in mutation accumulation in Daphnia pulex. Genome Biol 2010, 11:R46.

38. Wicker T, Sabot F, Hua-Van A, Bennetzen JL, Capy P, Chalhoub B, Flavell A, Leroy P, Morgante M, Panaud O, Paux E, SanMiguel P, Schulman AH: A unified classification system for eukaryotic transposable elements. Nat Rev Genet 2007. 8:973-982.

39. Penton EH, Sullender BW, Crease TJ: Pokey, a new DNA transposon in Daphnia (Cladocera: Crustacea). J Mol Evol 2002, 55:664-673.

40. Elliott TA: The activity and evolution of the Daphnia DNA transposon Pokey. MSc. Thesis. University of Guelph, Department of Integrative Biology; 2011.

41. Valizadeh P, Crease T: The association between breeding system and transposable element dynamics in Daphnia pulex. J Mol Evol 2008, 66:643-654.

42. Sullender BW, Crease TJ: The behavior of a Daphnia pulex transposable element in cyclically and obligately parthenogenetic populations. J Mol Evol 2001, 53:63-69.

43. Eickbush TH, Eickbush DG: Finely orchestrated movements: evolution of the ribosomal RNA genes. Genetics 2007, 175:477-485

44. Hebert PDN, Crease TJ: Clonal coexistence in Daphnia pulex (Leydig): another planktonic paradox. Science 1980, 207:1363-1365.

45. Hall TA: BioEdit: a user-friendly biological sequence alignment editor and analysis program for Windows 95/98/NT. Nucleic Acids Symp Ser 1999, 41:95-98.

46. Bruen TC, Philippe H, Bryant D: A simple and robust statistical test to detect the presence of recombination. Genetics 2006, 172:2665-2681.

47. Huson DH, Bryant D: Application of phylogenetic networks in evolutionary studies. Mol Biol Evol 2006, 23:254-267.

48. Kosakovsky Pond SL, Posada D, Gravenor MB, Woelk CH, Frost SDW: GARD: a genetic algorithm for recombination detection. Bioinformatics 2006, 22:3096-3098.

49. Hudson RR, Kaplan NL: Statistical properties of the number of recombination events in the history of a sample of dna sequences. Genetics 1985, 111:147-164

50. Librado P, Rozas J: DnaSP v5: A software for comprehensive analysis of DNA polymorphism data. Bioinformatics 2009, 25:1451-1452.

51. Lyngsø $R$, Song $Y$, Hein J: Minimum recombination histories by branch and bound. In Algorithms in Bioinformatics. Volume 3692. Edited by Casadio R, Myers G. Berlin/Heidelberg: Springer; 2005:239-250 [Lecture Notes in Computer Science.].

52. Song YS, Hein J: Constructing minimal ancestral recombination graphs. J Comput Biol 2005, 12:147-169.

53. Kuhner MK: Coalescent genealogy samplers: windows into population history. Trends Ecology Evol 2009, 24:86-93.

54. Tamura K, Peterson D, Peterson N, Stecher G, Nei M, Kumar S: MEGA5: Molecular Evolutionary Genetics Analysis using maximum likelihood, evolutionary distance, and maximum parsimony methods. Mol Biol Evol 2011, 28:2731-2739

55. Geyer CJ: Estimating normalizing constants and reweighting mixtures in Markov chain Monte Carlo. Technical Report No. 568. Minneapolis: School of Statistics, University of Minnesota; 1991 http://purl.umn.edu/58433.

56. Posada D: Evaluation of methods for detecting recombination from DNA sequences: empirical data. Mol Biol Evol 2002, 19:708-717.

57. Posada D, Crandall KA: Evaluation of methods for detecting recombination from DNA sequences: computer simulations. Proc Natl Acad Sci U S A 2001, 98:13757-13762.

58. Posada D, Crandall KA, Holmes EC: Recombination in evolutionary genomics. Annu Rev Genet 2002, 36:75-97. 
59. Graham J, McNeney B, Seillier-Moiseiwitsch F: Stepwise detection of recombination breakpoints in sequence alignments. Bioinformatics 2005 21:589-595.

60. Maynard Smith J: Analyzing the mosaic structure of genes. J Mol Evol 1992, 34:126-129.

61. Saitou N, Nei M: The neighbor-joining method: a new method for reconstructing phylogenetic trees. Mol Biol Evol 1987, 4:406-425.

62. Song YS, Hein J: Parsimonious reconstruction of sequence evolution and haplotype blocks. In Algorithms in Bioinformatics. Volume 2812. Edited by Benson G, Page R. Berlin/Heidelberg: Springer; 2003:287-302. Lecture Notes in Computer Science.

63. Qiu X, Wu L, Huang H, McDonel PE, Palumbo AV, Tiedje JM, Zhou J: Evaluation of PCR-generated chimeras, mutations, and heteroduplexes with 16S rRNA gene-based cloning. Appl Environ Microbiol 2001, 67:880-887.

64. Crease T, Floyd R, Cristescu M, Innes D: Evolutionary factors affecting lactate dehydrogenase $A$ and $B$ variation in the Daphnia pulex species complex. BMC Evol Biol 2011, 11:212.

65. McTaggart SJ, Dudycha JL, Omilian A, Crease TJ: Rates of recombination in the ribosomal DNA of apomictically propagated Daphnia obtusa lines. Genetics 2007, 175:311-320.

66. Omilian AR, Lynch M: Patterns of intraspecific DNA variation in the Daphnia nuclear genome. Genetics 2009, 182:325-336.

67. Bafna $V$, Bansal V: The number of recombination events in a sample history: conflict graph and lower bounds. IEEE/ACM Trans Comput Biol Bioinformatics 2004, 1:78-90.

68. Myers SR, Griffiths RC: Bounds on the minimum number of recombination events in a sample history. Genetics 2003, 163:375-394.

69. Glass SK, Moszczynska A, Crease TJ: The effect of transposon Pokey insertions on sequence variation in the 28S rRNA gene of Daphnia pulex. Genome 2008, 51:988-1000.

70. Carr M, Soloway J, Robinson T, Brookfield J: Mechanisms regulating the copy numbers of six LTR retrotransposons in the genome of Drosophila melanogaster. Chromosoma 2002, 110:511-518.

71. Duret L, Marais G, Biemont C: Transposons but not retrotransposons are located preferentially in regions of high recombination rate in Caenorhabditis elegans. Genetics 2000, 156:1661-1669.

72. Langley $\mathrm{CH}$, Montgomery E, Hudson R, Kaplan N, Charlesworth B: On the role of unequal exchange in the containment of transposable element copy number. Genet Res 1988, 52:223-235.

73. Rizzon C, Martin E, Marais G, Duret L, Segalat L, Biemont C: Patterns of selection against transposons inferred from the distribution of Tc1, Tc3 and Tc5 insertions in the mut-7 line of the nematode Caenorhabditis elegans. Genetics 2003, 165:1127-1135.

74. Schaack S, Pritham EJ, Wolf A, Lynch M: DNA transposon dynamics in populations of Daphnia pulex with and without sex. Proc $R$ Soc B Biol Sci 2010, 277:2381-2387.

75. Brookfield J: Models of repression of transposition in P-M hybrid dysgenesis by $\mathrm{P}$ cytotype and by zygotically encoded repressor proteins. Genetics 1991, 128:471-486.

76. Finnegan DJ: Transposable elements. In The Genome of Drosophila melanogaster. Volume viii. Edited by Lindsley DL, Zimm GG. New York: Academic: 1992:1096-1107.

77. Montgomery E, Charlesworth B, Langley $\mathrm{CH}$ : A test for the role of natural selection in the stabilization of transposable element copy number in a population of Drosophila melanogaster. Genet Res 1987, 49:31-41.

78. Biémont C: Population genetics of transposable DNA elements. Genetica 1992, 86:67-84

79. Bartolomé C, Maside $X$, Charlesworth B: On the abundance and distribution of transposable elements in the genome of Drosophila melanogaster. Mol Biol Evol 2002, 19:926-937.

80. Kim JM, Vanguri S, Boeke JD, Gabriel A, Voytas DF: Transposable elements and genome organization: a comprehensive survey of retrotransposons revealed by the complete Saccharomyces cerevisiae genome sequence. Genome Res 1998, 8:464-478.

81. Rizzon C, Marais G, Gouy M, Biemont C: Recombination rate and the distribution of transposable elements in the Drosophila melanogaster genome. Genome Res 2002, 12:400-407.

82. Wright SI, Agrawal N, Bureau TE: Effects of recombination rate and gene density on transposable element distributions in Arabidopsis thaliana. Genome Res 2003, 13:1897-1903.
83. Bao W, Jurka J: Origin and evolution of LINE-1 derived "half-L1" retrotransposons (HAL1). Gene 2010, 465:9-16.

84. Bleykasten-Grosshans C, Jung PP, Fritsch ES, Potier S, de Montigny J, Souciet JL: The Ty1 LTR-retrotransposon population in Saccharomyces cerevisiae genome: dynamics and sequence variations during mobility. FEMS Yeast Res 2011, 11:334-344.

85. Fischer SEJ, Wienholds E, Plasterk RHA: Continuous Exchange of Sequence Information Between Dispersed Tc1 Transposons in the Caenorhabditis elegans Genome. Genetics 2003, 164:127-134.

86. Jordan IK, McDonald JF: Evidence for the role of recombination in the regulatory evolution of Saccharomyces cerevisiae Ty elements. J Mol Evol 1998, 47:14-20.

87. Novick PA, Smith JD, Floumanhaft M, Ray DA, Boissinot S: The evolution and diversity of DNA transposons in the genome of the lizard Anolis carolinensis. Genome Biol Evol 2011, 3:1-14.

88. Keith JH, Schaeper CA, Fraser TS, Fraser MJ: Mutational analysis of highly conserved aspartate residues essential to the catalytic core of the piggyBac transposase. BMC Mol Biol 2008, 9:73.

89. Mitra R, Fain-Thornton J, Craig NL: piggyBac can bypass DNA synthesis during cut and paste transposition. EMBO J 2008, 27:1097-1109.

90. Zhang $\mathrm{X}$, Eickbush MT, Eickbush TH: Role of recombination in the long-term retention of transposable elements in rRNA gene loci. Genetics 2008, 180:1617-1626.

doi:10.1186/1759-8753-4-7

Cite this article as: Vergilino et al: Evolution of a transposon in Daphnia hybrid genomes. Mobile DNA 2013 4:7.

\section{Submit your next manuscript to BioMed Central and take full advantage of:}

- Convenient online submission

- Thorough peer review

- No space constraints or color figure charges

- Immediate publication on acceptance

- Inclusion in PubMed, CAS, Scopus and Google Scholar

- Research which is freely available for redistribution 